\title{
Combined surgery for lipomatous hypertrophy of the interatrial septum and pulmonary carcinoma
}

\author{
Nicolas Noiseux, MD, Pasquale Ferraro, MD, Guy Cousineau, MD, Ignacio Prieto, MD, and André Duranceau, MD, \\ Montreal, Quebec, Canada
}

From the Divisions of Thoracic and Cardiac Surgery and Department of Radiology, Centre Hospitalier de l'Université de Montréal, Montréal, Québec, Canada.

Received for publication Aug 20, 2001; accepted for publication Aug 28, 2001.

Address for reprints: Pasquale Ferraro, MD, Division of Thoracic Surgery, Hôpital Notre Dame, 1560 Sherbrooke St East, Montréal, Québec, Canada H2L 4M1 (E-mail: pasquale.ferraro@umontreal.ca).

J Thorac Cardiovasc Surg 2002;123:564-6

Copyright $\odot 2002$ by The American Association for Thoracic Surgery

$0022-5223 / 2002 \$ 35.00+0 \quad \mathbf{1 2 / 5 4 / 1 2 0 7 2 0}$

doi: $10.1067 / \mathrm{mtc} .2002 .120720$

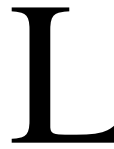

ipomatous hypertrophy of the interatrial septum (LHIS) is a rare benign tumor of the heart consisting of a nonencapsulated accumulation of mature fat, multivacuolated adipose cells, and enlarged cardiac myocytes. ${ }^{1-3}$ We report herein a case investigated for lung cancer in which LHIS was found incidentally. The cardiac tumor presented as a noncharacteristic form of LHIS with a pseudotumoral growth pattern. The cardiac and pulmonary tumors were removed through a right thoracotomy.

\section{Clinical Summary}

A 59-year-old woman was evaluated for dyspnea on exertion and increased sputum production. Results of the chest and cardiac examinations and electrocardiogram were unremarkable. The 


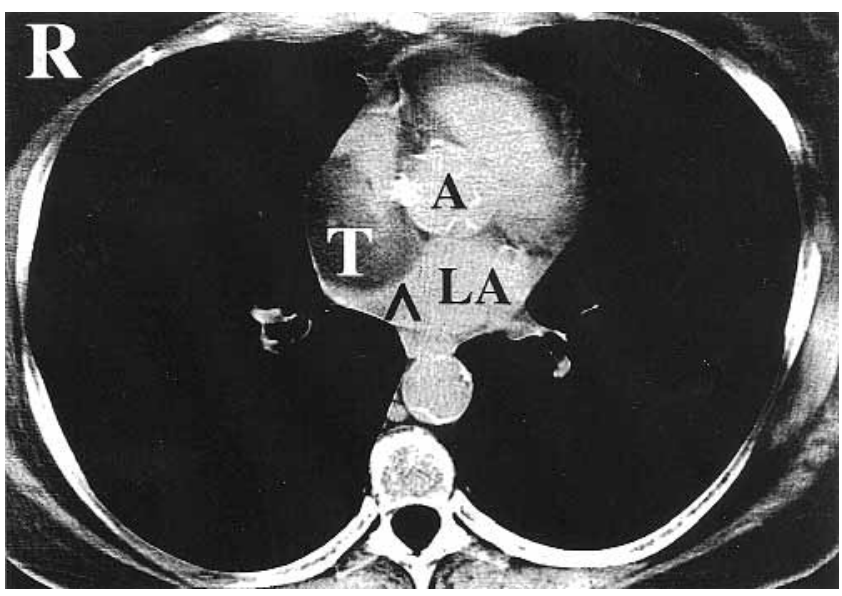

Figure 1. CT scan showing the cardiac tumor $(T)$ in the right atrium and relationship with other cardiac structures. $A$, Aorta; $L A$, left atrium; $\wedge$, atrial septum.

chest X-ray film and computed tomographic (CT) scan revealed an ill-defined $3.5-\mathrm{cm}$ mass in the posterior segment of the right upper lobe with slightly enlarged ipsilateral mediastinal lymph nodes. Also, a $4 \times 5 \times 7-\mathrm{cm}$ mass consisting of adipose tissue was found posteriorly in the right atrium near the superior vena cava junction (Figure 1). Transthoracic echocardiography revealed a large, globular echogenic mass of $18 \mathrm{~cm}^{2}$ (surface area) in the right atrium extending toward the superior vena cava. The mass had a broadbased attachment to the interatrial posterior septum. The rest of the septum was $10-\mathrm{mm}$ thick and infiltrated by adipose tissue. The fossa ovalis was normal. Thoracic magnetic resonance imaging confirmed the presence of the cardiac tumor in the interatrial septum (Figure 2). Bronchoscopic examination showed no endoluminal lesion but cytologic studies were positive for a non-small cell lung carcinoma. The metastatic workup showed no abnormalities, and pulmonary function tests showed adequate function.

Inasmuch as the tumor was large, the diagnosis of LHIS was considered less probable and the cardiac tumor was thought to be either a lipoma or liposarcoma. We thus decided to proceed with an exploration and possible excision of both the pulmonary and cardiac lesions through a right anterolateral thoracotomy. On opening the pericardium, we found a large yellowish tumor with no obvious capsule between the atria, with partial superior vena caval compression. Cardiopulmonary bypass was performed with ascending aortic perfusion and bicaval venous return. The right atrium was opened and the dissection continued around the mass, with excision of a portion of the right and left atria and the involved interatrial posterior septum. The deficit was reconstructed with an autologous pericardial patch. After successful weaning from cardiopulmonary bypass, a standard right upper lobectomy and mediastinal lymph node dissection were carried out.

Histologically, the cardiac tumor was characterized by a mixture of mature fat cells and multivacuolated cells, resembling brown fetal fat cells. Trapped myocardial fibers were also found, showing hypertrophy with large, somewhat polymorphic star-shaped nuclei (Figure 3 ). Electronic microscopic and immunohistochemical analysis con-

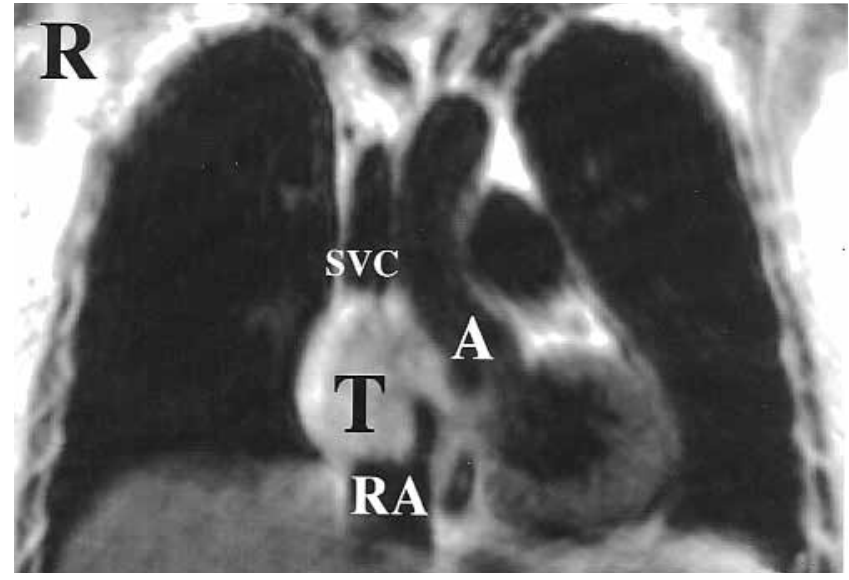

Figure 2. T1-weighted coronal section of magnetic resonance image showing the cardiac tumor $(T)$ in the right atrium $(R A)$ having the same density as fatty tissue. SVC, Superior vena cava; $A$, aorta.

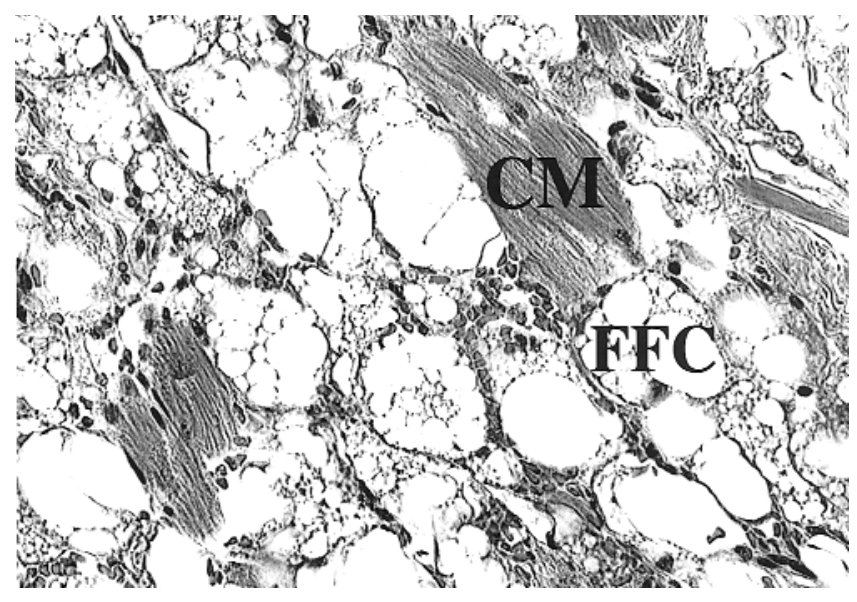

Figure 3. Gross photomicrograph of cardiac tumor showing multivacuolated fetal fat cells (FFC) and some trapped myocytes (CM). Hematoxylin and eosin staining at $400 \times$ magnification.

firmed the diagnosis of LHIS. The lung tumor was a grade III squamous cell carcinoma with no lymph node involvement and negative resection margins (stage T2 N0 [IB]).

Postoperatively, a slow junctional rhythm persisted, necessitating a permanent endocavitary AAI pacemaker. The patient otherwise recovered uneventfully and was discharged 17 days after the operation. At $2 \frac{1}{2}$ years' follow-up, the patient is doing well with no evidence of recurrent disease.

\section{Comment}

LHIS was first described by Prior $^{2}$ in 1964 . The great majority of early cases were incidentally found at autopsy. The first in vivo diagnosis of LHIS was not made until 1982 by Isner and associates. ${ }^{4}$ In its typical form, LHIS is characterized by an echo-dense globular thickening of the septum (>15 mm), sparing the fossa ovalis and giv- 
ing the characteristic sandglass appearance. LHIS is often associated with cardiac arrhythmias and sudden death, but most patients remain asymptomatic. ${ }^{3,5,6}$ The best form of treatment for LHIS remains controversial. Some authors suggest that asymptomatic LHIS detected by chance should not be surgically corrected. ${ }^{5,7,8}$

We report a case in which LHIS was found during investigation for lung cancer. The tumor was initially believed to be a liposarcoma or a benign lipoma. The decision to proceed with surgical removal was based on the large size of the tumor, its location, and compression of the interatrial septum. The right thoracotomy provided excellent exposure of the mediastinum, lung, great vessels, and right atrium and thus permitted excision of both lesions.

In summary, we present a rare atypical form of LHIS with a pseudotumoral growth pattern. In the presence of a large, globular, echogenic mass of adipose tissue in the interatrial septum, other features of LHIS must be sought. These features include thickening of the interatrial septum with a sandglass appearance and a normal fossa ovalis. LHIS discovered by chance should be considered for resection if there is evidence of obstruction or compression of neighboring structures or doubt as to the neoplastic nature of the tumor exists.

\section{References}

1. Burke A, Virmani R. Tumor of the heart and great vessels. Atlas of tumor pathology, 3rd series. Fascicule 16. Washington (DC): Armed Forces Institute of Pathology; 1996. p. 91-4.

2. Prior JT. Lipomatous hypertrophy of cardiac interatrial septum: a lesion resembling hibernoma, lipoblastomatosis and infiltrating lipoma. Arch Pathol. 1964;78:11-5.

3. Shirani J, Roberts WC. Clinical, electrocardiographic and morphologic features of massive fatty deposit (lipomatous hypertrophy) in the atrial septum. J Am Coll Cardiol. 1993;22:226-38.

4. Isner JM, Swan CS II, Mikus P, Carter B. Lipomatous hypertrophy of the interatrial septum: in vivo diagnosis. Circulation. 1982;66:470-3.

5. Zeebregts CJAM, Hensens AG, Timmermans J, Pruszczynski MS, Lacquet LK. Lipomatous hypertrophy of the interatrial septum: Indication for surgery? Eur J Cardiothorac Surg. 1997;11:785-7.

6. Fyke FE, Tajik AJ, Edwards WD, Seward JB. Diagnosis of lipomatous hypertrophy of the atrial septum by two-dimensional echocardiography. J Am Coll Cardiol. 1983;1:1352-7.

7. Alcocer JJ, Katz WE, Hattler BG. Surgical treatment of lipomatous hypertrophy of the interatrial septum. Ann Thorac Surg. 1998;65: 1784-6.

8. Oxorn DC, Edelist G, Goldman BS, Joyner CD. Echocardiography and excision of lipomatous hypertrophy of the interatrial septum. Ann Thorac Surg. 1999;67:852-4. 